\begin{tabular}{|c|c|c|}
\hline & Int.J.Curr.Microbiol.App.Sci (2016) 5(10): 128-134 & \multirow{2}{*}{ 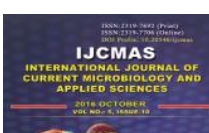 } \\
\hline & \multirow{4}{*}{$\begin{array}{l}\text { International Journal of Current Microbiology and Applied Sciences } \\
\text { ISSN: 2319-7706 Volume } 5 \text { Number } 10 \text { (2016) pp. 128-134 } \\
\text { Journal homepage: http://www.ijcmas.com }\end{array}$} & \\
\hline & & \\
\hline EXCELLENT & & \\
\hline PUBLISHERS & & wwwi.jicmas.com \\
\hline
\end{tabular}

Original Research Article

http://dx.doi.org/10.20546/ijcmas.2016.510.015

\title{
Prevalence and Antibiotic Susceptibility Pattern of Escherichia coli Positive Urinary Tract Infections in a Rural Tertiary Care Hospital in Rohtas, Bihar, India
}

\author{
Rana Pratap ${ }^{1 *}$, Amit Kumar ${ }^{2}$ and Ahmad Nadeem Aslami ${ }^{3}$ \\ ${ }^{1}$ Department of Microbiology, NMCH, Sasaram, India \\ ${ }^{2}$ Department of Pharmacology, NMCH, Sasaram, India \\ ${ }^{3}$ Department of Community Medicine, NMCH, Sasaram, India \\ *Corresponding author email id:
}

\section{Keywords \\ Antibiotic \\ Susceptibility \\ Pattern, \\ Escherichia coli \\ Urinary Tract \\ Infections.}

\begin{tabular}{l}
\hline Article Info \\
\hline Accepted: \\
12 September 2016 \\
Available Online: \\
10 October 2016
\end{tabular}

\section{A B S T R A C T}

Urinary tract infection (UTI) is a common condition affecting all age groups. E.coli and several other uropathogens account for most UTIC cases. Although definitive diagnosis is based on culture reports, most of the UTI are treated empirically. Multidrug Resistance (MDR) cases of UTI is also on the rise. A cross sectional study was conducted to find out the prevalence of E.coli positive UTI cases and to know its susceptibility pattern against commonly used antibiotics. Urine samples were collected from patients attending OPD's who were suspected to be having UTI. Identification of organism by urine culture and susceptibility pattern was based on standard conventional bacteriological techniques using Kirby-Bauer disc diffusion method. Clinical and Laboratory Standard Institute (CLSI) guidelines were followed. $64.57 \%$ isolates were positive for E.coli. There were $56.6 \%$ females and $43.4 \%$ males. $44.2 \%$ of patients with E.coli positive UTI were found within the age range of 20-39 years. E.coli exhibited highest resistance to Nalidixic acid. Amoxycillin, Cefixime, Cotrimoxazole, Ceftriaxone and Ofloxacin also showed high resistance. 87.6\% E.coli were MDR cases which may be attributed to their prevailing usage and abuse in the area under study. Empirical treatment should be carefully selected based upon the sensitivity data of uropathogens of that particular area. Frequent monitoring of antimicrobial susceptibility for uropathogens in the community and hospital setting is recommended.

\section{Introduction}

Urinary tract infection (UTI) is a very common condition and a major cause of seeking medical consultation (Omoregie et al., 2008). UTI are also prevalent in community in all age groups and requires urgent treatment (Orrett et al., 2006). Most of the UTI cases are treated on an empirical basis of geographical distribution of antibiotic susceptibility in absence of any laboratory investigations (Bercion et al., 2009). UTI can be caused by several uropathogens. In developing countries like India, E.coli accounts for most of the UTI cases across all age groups, in both sexes with variable clinical presentation ranging from mild asymptotic cases to severe complicated UTI. 
Diagnosis and definitive treatment of UTI is mainly based on bacteriological culture and antibiotic sensitivity. In past decade, indiscriminate use of antibiotics resulted in worldwide rise of Multidrug Resistance (MDR) cases (Davies et al., 2010).

Antibiotic resistant uropathogens makes empirical treatment of UTI even more difficult. Hence analysing antibiotic susceptibility pattern of uropathogens will help to overcome the therapeutic dilemmas and guide in selection of appropriate antibiotics for empirical treatment. The aim of this study was find out the prevalence, age and sex distribution of E.coli positive UTI in this area and to know its susceptibility pattern against commonly used antibiotics.

\section{Materials and Methods}

This hospital based cross-sectional study was conducted in Department of Microbiology at Narayan Medical College \& Hospital, situated in a rural area of Sasaram, Bihar in collaboration with the Department of Community Medicine and Pharmacology. The study period was from June 2016 to august 2016. The study was approved by Institutional Ethical Committee. Urine samples were collected from patients attending OPD's who were suspected to be having UTI after getting their consent.

Clean-catch midstream urine was collected into a wide mouth sterile universal container. A proper instruction was given to the patients regarding the method of collection. Males were asked to retract prepuce, cleanse the glans with soap and water and then collect the sample. Females were instructed to thoroughly clean the anogenital area from front to back, pass urine with labia separated and collect sample. Later labelled specimens with patient's age and sex on container were transported to the laboratory.

Inadequate urine samples $(<10 \mathrm{~mL})$, urine bag collected samples, specimens collected more than 2 hours before submission, specimens in leaking, or dirty unsterile containers and specimens revealing growth of more than two types of bacteria on culture were excluded from the study. Patients on long term antibiotic therapy prior to or during the investigation were also excluded.

Urine culture was done by standard loop method, a semi-quantitative method. Before processing urine is mixed well and a loopfull $(0.001 \mathrm{ml})$ urine was streaked onto the surface of blood agar and Mac Conkey's agar. These plates were incubated aerobically at $37^{\circ} \mathrm{C}$ FOR $18-24$ hours. Bacterial counts were expressed in colony forming units (CFU) per millilitre $(\mathrm{mL})$. A count of $\geq 10^{5} \mathrm{CFU} / \mathrm{mL}$ was considered significant bacteriuria which conferred to "Kass concept of significant bacteriuria". Identification of organism was based on standard conventional bacteriological techniques (Forbes et al., 2002).

Antimicrobial susceptibility testing was performed as per Kirby-Bauer disc diffusion method as per guidelines. The entire disc was obtained from Hi Media Laboratories, India. Quality controls were regularly checked by standard strains of E.coli ATCC 25922. Clinical and Laboratory Standard Institute (CLSI) guidelines were followed to interpret sensitivity and resistance on the basis of the diameters of zones of inhibition of bacterial growth as recommended by the disc manufacturer (Clinical and Laboratory Standard Institute, 2013).

Antibiotics against which sensitivity was tested in the present study included Amoxycillin, Cefixime, Ceftriaxone, Amikacin, Tobramycin, Nalidixic acid, 
Ciprofloxacin, Ofloxacin, Levofloxacin, Azithromycin and Cotrimoxazole. The concentrations at which they were used is given in Table 2. An isolate was considered as MDR if found resistant o three or more antimicrobials to different classes/ groups of antimicrobials (http://ecdc.europa.eu/en/activities/diseasepr ogrammes/ARHAI/Documents/table-

3Clinical_Microbiology_and_Infection.pdf).

Data was entered in MS Excel and analyzed by the statistical package SPSS software, version 16.0. Descriptive and inferential statistics were applied (percentages and $\mathrm{Z}$ test). The values of $p$ were kept significant at the level of 0.05 .

\section{Results and Discussion}

Bacteriological aetiology of UTI in patients of this hospital was determined in 538 urine samples. Bacteria were successfully isolated from 175 (32.5\%; 175/538) samples. Out of 175 bacterial isolates, 113 (64.57\%) isolates were positive for E.coli. Thus E.coli was most common isolates, followed by Klebsiella pneumonia (13.71\%), Pseudomonas aeruginosa (10.86\%), Proteus spp. (5.14\%), Acinatobacter baumanii (2.85\%), Staphylococcal aureus $(1.71 \%)$ and Candida spp.(1.16\%).

Among E.coli positive UTI cases, there were more females $(56.6 \%, 64 / 113)$ than males. $(43.4 \%, 49 / 113)$ and this difference was statistically significant $\quad(\mathrm{Z}=\quad-1.996$; $p=0.0459$ ). The incidence of UTI was ranged in patients between 4-80 years old. The highest number $(50 ; 44.2 \%)$ of patients with E.coli positive UTI were found within the age range of 20-39 years. In this age group, proportion of females $(72.0 \%$; 36/50) was significantly more as compared to males (28.0\%; 14/50).

In comparison, E.coli isolates were found less in age group $\leq 19$ years and $\geq 60$ years.
Considering the overall incidence among all age groups, females of age group 20-39 years $(56.25 \%$; 36/64) and males of $40-59$ years $(42.86 \% ; 21 / 49)$ were more affected (Table 1).

Antibiotic resistant pattern of E.coli against a number of antibiotics was also tested out. E.coli exhibited highest resistance to Nalidixic acid (93.6\%) followed by Amoxycillin (91.1\%), Cefixime (77.9\%), Cotrimoxazole $(76.1 \%)$, Ceftriaxone and Ofloxacin (69.9\%). Out of 113 E.coli, 99 $(87.6 \%)$ were multidrug resistance (MDR) as the isolates were non-susceptible to at least 1 agent in $\geq 3$ antimicrobial categories. (Table 2)

The culture positive rate in this study was $32.5 \%$. A similar rate was observed in other studies. In this study we have excluded sterile samples, samples showing insignificant growth $\left(<10^{5} \mathrm{CFU} / \mathrm{mL}\right)$ and samples contaminated with polymicrobial growth of more than three types of organism (Arjunan et al., 2010; Taneja et al., 2010; Singhal et al., 2014).

The most common uropathogen in our study was E.coli, found in $65 \%$ isolates. Similar pattern was seen in study done by other studies (Singhal et al., 2014; Kamat et al., 2009). The second most common uropathogen in this study was Klebsiella pneumonia, followed by Pseudomonas aeruginosa and Proteus spp. (Manikandan et al., 2014).

Infection frequency of Acinatobacter baumanii, Staphylococcal aureus and Candida spp. was found less in this study in comparison to E.coli. While most other studies have reported the involvement of Enterobacter spp. and enterococcus faecalis as one of the common pathogen in UTI cases, our study in this area has not shown any such finding (Niranjan et al., 2014). 
The etio-pathological variation in UTI cases may be due to differences in host factors, environmental factors, health care and hygiene practices and socio-economic conditions in different part of the world. In this study, we have described the sociodemographic factors, prevalence of antibiotic susceptibility and resistance in patients of UTI predominantly caused by E.coli. The sex distribution of the UTI patients in this study is similar to other reported studies showing a predominance of females $(56.6 \%)$.

The increased incidence of UTI among females is related to the anatomical differences between male and female genitourinary symptoms and alteration in normal vaginal flora. Among females, more cases were seen in reproductive age group because of their increase sexual activities, for example "honeymoon cystitis" and "Pyelitis of Pregnancy".

In this study, males of 40-59 years were more affected which may be related to the increased incidence of bladder outlet obstruction such as prostitis and bladder stone. E.coli isolates were found less at the extremes of ages in this study probably because of their dependence for consultation in this tertiary care centre.

The most effective antimicrobial agents in this study were Amino glycosides (Amikacin, Tobramycin), followed by Fluoroquinolone (Levofloxacin) and Macrolide (Azithromycin). In this study Amikacin is the most effective antimicrobial against E.coli. Our result is further supported by different authors (Shalini et al., 2011).

E.coli showed considerable resistance to Amoxycillin and Nalidyxic acid $>90 \%$ in both) followed by Cefixime and Cotrimoxazole. It is important to note that the E.coli isolates showed considerable resistance to beta-lactam antibiotics probably because they belonged to Extended spectrum beta lactamase (ESBL) group. Another important finding is the resistance to most of the older generation fluoroquinolones and sensitive to only levofloxacin.

The rising resistance for fluoroquinolones is based on several factors (Hooton, 2003). Recent guidelines from the Infectious Diseases Society of America recommended that empiric antibiotic therapy for UTIs should be based on local resistance data, drug availability, and antibiotic intolerance/allergy history of treated patients (Gupta et al., 2011).

Fluoroquinolones

(Ciprofloxacin, Ofloxacin) and Cotrimoxazole are most recommended antimicrobials for empirical treatment of UTI in developing countries like India. But in this study we found the resistance against both these groups which reflects the geographical variation of the sensitivity data. Hence based upon the data of this study for our setting in this zone, the recommended empirical therapy before culture and sensitivity will be amino glycosides like Amikacin, Levofloxacin and/ or Azithromycin.

However, in older patients amikacin should be used carefully due to risk of ototoxicity and nephrotoxicity. ${ }^{21} 87.6 \%$ isolates were MDR E.coli.

It could be due to overall increase in prevalence of MDR pathogens in the community, which is associated with the injudicious irrational use of available antibiotics as well as newer molecules and improper personal hygiene by health staff and patients. 
Table.1 Distribution of UTI patients according to age groups and gender.

\begin{tabular}{|l|c|c|c|c|c|c|}
\hline Age Groups & Males (\%) & Females (\%) & Total & \%of Total & Z & $p$ \\
\hline$\leq 19$ yrs & $7(58.4 \%)$ & $5(41.6 \%)$ & 12 & $10.6 \%$ & 0.8 & 0.410 \\
\hline 20- 39 yrs & $14(28.0 \%)$ & $36(72.0 \%)$ & 50 & $44.2 \%$ & 4.4 & $<0.0001$ \\
\hline 40- 59 yrs & $21(61.7 \%)$ & $13(38.3 \%)$ & 34 & $30.1 \%$ & 1.9 & 0.0537 \\
\hline$\geq 60$ yrs & $7(41.2 \%)$ & $10(58.8 \%)$ & 17 & $15.1 \%$ & 1 & 0.304 \\
\hline Total & $49(43.4 \%)$ & $64(56.6 \%)$ & 113 & $100 \%$ & 2 & 0.0472 \\
\hline \multicolumn{7}{|c|}{$\chi^{2}=10.6 ; d f=3 ; p=0.014$} \\
\hline
\end{tabular}

Table.2 Antibiotic sensitivity pattern of E.coli isolates

\begin{tabular}{|c|c|c|c|c|c|c|c|c|c|}
\hline \multirow[t]{2}{*}{ Antibiotics } & \multirow[t]{2}{*}{ Codes } & \multirow{2}{*}{$\begin{array}{c}\text { Disc } \\
\text { content } \\
(\mu \mathrm{g})\end{array}$} & \multirow{2}{*}{$\begin{array}{c}\text { Diffusion } \\
\text { zone } \\
\text { breakpoint } \\
\text { (mm) }\end{array}$} & \multicolumn{2}{|c|}{ Sensitive } & \multicolumn{2}{|c|}{$\begin{array}{c}\text { Intermedi } \\
\text { ate }\end{array}$} & \multicolumn{2}{|c|}{ Resistant } \\
\hline & & & & $\mathbf{n}$ & $\%$ & $\mathbf{N}$ & $\%$ & $\mathbf{n}$ & $\%$ \\
\hline Amoxycillin & AMX & 30 & $\leq 14$ & 7 & 6.2 & 3 & 2.7 & 103 & 91.1 \\
\hline Cefixime & CFM & 5 & $\leq 15$ & 20 & 17.7 & 5 & 4.4 & 88 & 77.9 \\
\hline Ceftriaxone & CTR & 30 & $\leq 13$ & 23 & 20.4 & 11 & 9.7 & 79 & 69.9 \\
\hline Amikacin & AK & 30 & $\leq 14$ & 87 & 77.0 & 2 & 1.7 & 24 & 21.3 \\
\hline Tobramycin & TOB & 10 & $\leq 12$ & 80 & 70.8 & 9 & 8.0 & 24 & 21.2 \\
\hline Nalidixic Acid & NA & 30 & $\leq 13$ & 4 & 3.5 & 3 & 2.9 & 106 & 93.6 \\
\hline Ciprofloxacin & CIP & 5 & $\leq 15$ & 21 & 18.6 & 20 & 17.7 & 72 & 63.7 \\
\hline Ofloxacin & $\mathrm{OF}$ & 5 & $\leq 28$ & 18 & 16.0 & 16 & 14.1 & 79 & 69.9 \\
\hline Levofloxacin & LE & 5 & $\leq 29$ & 77 & 68.1 & 23 & 20.3 & 13 & 11.6 \\
\hline Azithromycin & AZM & 15 & $\leq 18$ & 72 & 63.7 & 8 & 7.1 & 33 & 29.2 \\
\hline Cotrimoxazole & $\mathrm{COT}$ & $\begin{array}{l}1.25 / \\
23.75\end{array}$ & $\leq 26$ & 23 & 20.3 & 4 & 3.6 & 86 & 76.1 \\
\hline
\end{tabular}

In conclusion, there is variation in antibiotic susceptibility pattern of E.coli positive UTI. Hence empirical treatment should be carefully selected based upon the susceptibility data of isolates of that particular area. Frequent monitoring of antimicrobial susceptibility for uropathogens in the community and hospital setting is recommended. To limit the increasing incidence of drug resistance, strict national 
and hospital antibiotic policies especially to the newer antibiotics, is the need of the hour.

\section{References}

Arjunan, M., Al-Salamah, A.A., Amuthan, M. 2010. Prevalence and antibiotic susceptibility of uropathogens in patients from a rural environment, Tamil Nadu. Am. J. Infect. Dis., 6: 293.

Avinash Laghawe, Apoorva Tripathi, S.B. Saxena. 2015. Aetiology of Urinary Tract Infection and antimicrobial susceptibility pattern of urinary isolates in tertiary care hospital in Central India: A retrospective analysis. Int. J. Curr. Microbiol. App. Sci., 4: 962-970.

Aypak, C., Altunsoy, A., Düzgün, N. 2009. Empiric antibiotic therapy in acute uncomplicated urinary tract infections and fluoroquinolone resistance: A prospective observational study. Ann. Clin. Microbiol. Antimicrob., 8: 27.

Bercion, R., Kpinde, D.M., Manirakiza, A. et al. 2009. Increasing prevalence of antimicrobial resistance among Enterobacteriaceae uropathogens in Bangui, Central African Republic. J. Infect. Develop.Countries, 3: 187-190.

Clinical and Laboratory Standard Institute. 2013. Performance standards for Antimicrobial Susceptibility Testing. Clinical and Laboratory Standard Institute, Document M100S23.http://ecdc.europa.eu/en/activities/ diseaseprogrammes/ARHAI/Documen ts/table-

3_Clinical_Microbiology_and_Infecti on.pdf

Das, R.N., Chandrashekhar, T.S., Joshi, H.S., Gurung, M., Shrestha, N., Shivananda, P.G. 2006. Frequency and susceptibility profile of pathogens causing urinary tract infections at a tertiary care hospital in western Nepal. Singapore Med. J., 47: 281285.

Davies, J., Davies, D. 2010.Origins and Evolution of Antibiotic Resistance. Microbiol. Mol. Biol. Rev., MMBR, 74: 417-433.

Forbes, B.A., Sahm, D.F. and Weissfeld, A.S. 2002. Infections of the Urinary tract, In; Baileys and Scott s (11thedt.) Diagnostic Microbiology St. Louis, Missouri, Mosby, 927-238.

Gupta, K., Hooton, T.M., Naber, K.G., et al. 2011. International clinical practice guidelines for the treatment of acute uncomplicated cystitis and pyelonephritis in women: a 2010 update by the infectious diseases society of America and the European society for microbiology and infectious diseases. Clin. Infect. Dis., 52: e103-e120.

Hooton, T.M. 2003. Fluoroquinolones and resistance in the treatment of uncomplicated urinary tract infection. Int. J. Antimicrob. Agents, 22: S65S72.

Kamat, U.S., Fereirra, A., Amonkar, D., Motghare, D.D., Kulkarni, M.S. 2009. Epidemiology of hospital acquired urinary tract infections in a medical college hospital in Goa. Indian $J$. Urol., 25: 76-80.

Khawcharoenporn, T., Vasoo, S., Singh, K. 2013. Urinary Tract Infections due to Multidrug-Resistant

Enterobacteriaceae: Prevalence and Risk Factors in a Chicago Emergency Department. Emergency Med. Int., 258517.

Manikandan, C. and Amsath, A. 2014. Antibiotic susceptibility pattern of Escherichia coli isolated from urine samples in Pattukkottai, Tamilnadu. Int. J. Curr. Microbiol. App. Sci., 3: 449-457. 
Niranjan, V., Malini, A. 2014. Antimicrobial resistance pattern in Escherichia coli causing urinary tract infection among inpatients. Indian J. Med. Res., 139: 945-8.

Omoregie, R., Erebor, J.O., Ahonkhai, I., Isobor, J.O., Ogefere, H.O. 2008. Observed changes in the prevalence of uropathogens in Benin City, Nigeria. N Z J. Med. Lab. Sci., 62: 29-31.

Orrett, F.A., Davis, G.K. 2006. A comparison of the antimicrobial susceptibility profile of urinary pathogens for the years 1999 and 2003. West Indian Med. J., 55: 95-99.

Prakash, D., Saxena, R.S. 2013. Distribution and Antimicrobial Susceptibility Pattern of Bacterial Pathogens Causing Urinary Tract Infection in
Urban Community of Meerut City, India. ISRN Microbiol., 749629.

Shalini, Joshi, M.C., Rashid, M.K., Joshi, H.S. 2011. Study of Antibiotic Sensitivity Pattern In Urinary Tract Infection At A Tertiary Hospital. NJIRM, 2: 43-6.

Singhal, A., Sharma, R., Jain, M., \& Vyas, L. Hospital and Community Isolates of Uropathogens and their Antibiotic Sensitivity Pattern from a Tertiary Care Hospital in North West India. Annals of Med. Health Sci. Res., 4: 51-56.

Taneja, N., Chatterjee, S.S., Singh, M., Singh, S., Sharma, M. 2010. Pediatric urinary tract infections in a tertiary care center from North India. Indian J. Med. Res., 131: 101-5.

\section{How to cite this article:}

Rana Pratap, Amit Kumar and Ahmad Nadeem Aslami. 2016. Prevalence and Antibiotic Susceptibility Pattern of Escherichia coli Positive Urinary Tract Infections in a Rural Tertiary Care Hospital in Rohtas, Bihar, India. Int.J.Curr.Microbiol.App.Sci. 5(10): 128-134. doi: http://dx.doi.org/10.20546/ijcmas.2016.510.015 